\title{
Corynebacterium Granulosum P40
}

National Cancer Institute

\section{Source}

National Cancer Institute. Corynebacterium Granulosum P40. NCI Thesaurus. Code C990.

An insoluble fraction isolated from the bacterium Corynebacterium granulosum with potential cancer immunotherapeutic activity. As a non-specific immunostimulant, Corynebacterium granulosum P40 activates the reticuloendothelial system, induces the production of certain cytokines, enhances macrophage activity, and potentiates a delayed-type hypersensitivity response when co-administered with an antigen. (NCI04) 